\title{
Vascular endothelial growth factor-A (VEGFA) gene polymorphisms may serve as genetic marker for Coronary Heart Disease (CHD)
}

\section{Kang Huang}

Central South University Xiangya School of Medicine Affiliated Haikou Hospital

Shijuan Lu ( $\sim$ lushijuan_361@163.com)

Central South University Xiangya School of Medicine Affiliated Haikou Hospital

Yilei Zhou

Central South University Xiangya School of Medicine Affiliated Haikou Hospital

Dehong Lin

Central South University Xiangya School of Medicine Affiliated Haikou Hospital

Zibin Chen

Central South University Xiangya School of Medicine Affiliated Haikou Hospital

Zanrui Zhong

Central South University Xiangya School of Medicine Affiliated Haikou Hospital

Jianghua Zhong

Central South University Xiangya School of Medicine Affiliated Haikou Hospital

\section{Research}

Keywords: VEGFA, coronary heart disease (CHD), susceptibility, polymorphism, case - control study

Posted Date: November 19th, 2020

DOI: https://doi.org/10.21203/rs.3.rs-109728/v1

License: (c) (i) This work is licensed under a Creative Commons Attribution 4.0 International License. Read Full License 


\begin{abstract}
Purpose: Coronary heart disease (CHD) is a common cardiovascular disease resulting from interaction of multiple environmental and genetic factors. This study aimed to confirm whether single nucleotide polymorphisms (SNPs) in VEGFA gene were associated with CHD in the Han Chinese population.
\end{abstract}

Materials and Methods: Blood samples were collected from 501 CHD patients and 496 healthy individuals. Genotyping of five SNPs within VEGFA was performed using Agena MassARRAY platform. Odd ratios (ORs) and $95 \%$ confidence intervals $(95 \% \mathrm{Cls})$ were calculated to evaluate the association between SNPs and CHD risk.

Results: The genotype "C/T" of rs3025021 in VEGFA was found to be associated with CHD susceptibility $(\mathrm{OR}=1.35,95 \% \mathrm{Cl}=1.02-1.80, p=0.038)$ in the overall. Rs833068 was observed to be associated with the reduced risk of CHD at age $>61$ years and $\leq 61$ years, respectively. And three loci (rs833068, rs3025021 and rs6905288) were related to the CHD risk in males. In addition, rs3025021 was associated with increased risk of CHD in patients with hypertension or diabetes. Conversely, three loci (rs833068, rs3025030 and rs6905288) were related to the CHD risk in patients without hypertension. The rs833068 was associated with reduced risk of CHD in patients without diabetes. Finally, we found a strong LD between rs833068

and rs833070.

Conclusion: Gene polymorphisms in VEGFA were notably correlated with altered CHD risk in the Han Chinese population. Large sample size and well designed studies are needed to further clarify the potential mechanisms underlying the CHD.

\title{
1. Introduction
}

Coronary artery disease (CAD) continues to be one of the most common cardiovascular disease with high morbidity and mortality (1), which represents a public health challenge in both industrialized and developing countries (2). Coronary heart disease (CHD) is the most common and severe manifestation of CAD (3). CHD, the myocardial functional or organic lesion, is caused by coronary artery stenosis or occlusion, or shortage of blood and oxygen supply (4). According to the data from the American Heart Association in 2009, cardiovascular disease has become the leading cause of death in the world (5). As China gradually enters an aging society and the number of the elderly gradually increases, CHD is the second leading cause of death after malignant tumors (6).

Numerous studies of its etiology and pathogenesis indicated that many factors have been proved to be associated with the occurrence of CHD, such as age, gender, diabetes, diet, and genetic factors $(7,8)$. Despite the specific factors influencing susceptibility of CHD is still unknown, recent molecular epidemiological studies have pointed to the potential and significant role of gene variants associated CHD, such as CCDC92 (9), AdipoQ (10). The vascular permeability factor (VEGF) gene, consisting of VEGFA, VEGFB, VEGFC, VEGFD, VEGFE, VEGFF and placental growth factor, is located on chromosome 6p21.3 and could express different isoforms of proteins (11). Usually, VEGFA is referred as VAGF. The human VEGFA gene is very polymorphic. Doxing Liu et al (12) showed that 3 SNPs (rs699947, rs3025039 and rs1570360) of VEGFA were remarkably associated with the susceptibility to CHD in a Han Chinese population. In addition, a systematic review and meta-analysis showed that VEGFA rs699947 polymorphism was not associated with CHD (13). However, there are few studies which are able to clarify the potential relationship between VEGFA genetic polymorphisms and the susceptibility to CHD in a Han Chinese population.

Therefore, a case-control study was carried out to evaluate the influence of VEGFA polymorphisms at allele, genotype, and haplotype interface with development of CHD among Han Chinese population.

\section{Materials And Methods}

\subsection{Study participants}

Using a case-control study, $501 \mathrm{CHD}$ patients and 496 unrelated healthy controls in a large cohort of Han Chinese population were recruited from Central South University Xiangya School of Medicine Affiliated Haikou Hospital to investigate whether the VEGFA variants have influence on CHD. All CHD patients were diagnosed including classic ischemic symptoms, plus one or more electrocardiographic (ECG) changes (ST-segment depression or elevation of $\geq$ $0.5 \mathrm{~mm}$, T-wave inversion of $\geq 3 \mathrm{~mm}$ in $\geq 3$ leads, or left bundle branch block), in addition to increased cardiac markers such as creatinine kinase-MB and troponin T (9). Patients with other diseases, such as complex hematologic, autoimmune, congenital cardiac structural or functional abnormalities, or tumors were excluded. At the same time, the 496 healthy controls were randomly selected from the same hospital in the same period and were diagnosed without heart disease or other diseases. All subjects were unrelated individuals and at least three generations of Han ancestors.

\subsection{Data collection}

The protocol of the present study was approved by the clinical investigative ethical committee of Central South University Xiangya School of Medicine Affiliated Haikou Hospital, and all procedures were performed in compliance with medical research involving humans of the World Medical Association Declaration of Helsinki. Informed consent forms were obtained from all participants after a full explanation. Blood samples from each individual were harvested respectively at the time of initial diagnosis. Subsequently, about $5 \mathrm{ml}$ venous blood samples were collected from each participant into tubes containing ethylenediamine tetraacetic acid (EDTA) for anticoagulation. A Whole Blood Genomic DNA Extraction Kit (Tiangen Biotech, Beijing, China) was used to extract genomic DNA from peripheral blood samples according to the manufacturer's instructions. And the purity and concentration of the DNA samples were evaluated with the NanoDrop $2000 \mathrm{C}$ (Thermo Scientifc, Waltham, Massachusetts, USA) (14). The isolated DNA samples were stored at - $80{ }^{\circ} \mathrm{C}$ until analysis.

\subsection{SNP selection and genotyping}


Five candidate SNPs (rs833068, rs833070, rs3025021, rs3025030, rs6905288) in the VEGFA gene were selected with the minor allele frequency (MAF) >0.05 in Han Chinese from the 1000 Genomes Project (http://www.1000genomes.org/). The primers for amplification and extension reactions were designed with Agena MassARRAY Assay Design 3.0 Software (Table 1) (15). The SNP genotyping was performed with Agena MassARRAY RS1000 according to the manufacturer's instruction. The data management and analysis were carried using Agena Typer 4.0 software $(15,16)$.

Table 1

PCR primer for this study.

\begin{tabular}{|c|c|c|c|c|}
\hline SNP-ID & Foward primer $\left(5^{\prime}-3^{\prime}\right)$ for PCR & Reverse primer $\left(5^{\prime}-3^{\prime}\right)$ for PCR & UEP_DIR & UEP SEQ \\
\hline rs833068 & ACGTTGGATGGGGAGAGTGGACATTTAGTG & ACGTTGGATGGAGATCCCATTAGGCTGAG & $\mathrm{R}$ & GCTCACACAGGAAGGGT \\
\hline rs833070 & ACGTTGGATGGCTCAGCCTAATGGGATCTC & ACGTTGGATGAGTTCACAGCACCCGAACAT & $\mathrm{R}$ & cattaACAGCACCCGAACATAGTCAA \\
\hline rs3025021 & ACGTTGGATGCACAGAGGCCTCCTTGCAG & ACGTTGGATGGGTGTGATGGGAGGCTAAG & $\mathrm{R}$ & CACAGCCTCCGACCC \\
\hline rs3025030 & ACGTTGGATGTGGCTGTTCGTTTAGGATGG & ACGTTGGATGGACTGGTGGAGGATTAAAGG & $\mathrm{R}$ & GGAGGATTAAAGGTATCTAGTATT \\
\hline rs6905288 & ACGTTGGATGTGAGGAATAATAACCACCCC & ACGTTGGATGAAGTAGAGAGAAGCCATCCC & $\mathrm{F}$ & GCCATCCСTGCCCCA \\
\hline
\end{tabular}

SNP, Single-nucleotide polymorphism; UEP SEQ, Unextended mini-sequencing primer; DIR: direction.

\subsection{Statistical analysis}

Statistical analyses were analyzed using SPSS 19.0 (SPSS, Chicago, IL, USA) and Microsoft Excel. The two-sided Chi-square tests and independent sample Student's t-test were applied to assess the differences in the distribution of gender and age between cases and controls, respectively. The genotype frequencies of the control group were tested for the Hardy-Weinberg equilibrium (HWE) using Chi-square test. Chi-squared test was used to calculate the allele and genotype frequencies of each SNP between cases and controls. Odds ratios (ORs) and 95\% confidence intervals (Cls) were calculated to estimate the association between VEGFA gene polymorphisms and the CHD risk using logistic regression analysis with or without adjustment for age and gender (17). The wild type allele was used as a reference. The four genetic models analyses were applied using PLINK software (Version 1.07) to evaluate the associations between SNPs and the CHD risk (18). Then, we conducted stratification analysis on age, gender, CHD-associated diseases. Finally, we performed linkage disequilibrium (LD) and haplotype analysis using the Haploview software package (version 4.2). All $p$ values of statistical tests in this study were two-sided, and $p<0.05$ indicated statistical significance.

\section{Results}

\subsection{Participant characteristics.}

We recruited $501 \mathrm{CHD}$ patients (mean age $61.32 \pm 11.70$ years old) consisting of 320 males and 181 females and 496 unrelated healthy individuals (mean age $60.69 \pm 6.42$ years old) containing of 318 healthy males and 178 females in this study. There were no statistically significant differences on the distribution of gender and age between the cases and controls $(p=0.885, p=0.289$, respectively). In the case group, there were 296 patients with hypertension and 101 patients with diabetes, while there were 205 patients without hypertension and 400 patients without diabetes, respectively. The basic characteristics of all subjects were shown in Table 2. 
Table 2

Characteristics of the cases and controls.

\begin{tabular}{|llll|}
\hline Variables & Cases $(\mathbf{n}=\mathbf{5 0 1})$ & Controls $(\mathbf{n}=\mathbf{4 9 6})$ & $\boldsymbol{p}$ \\
\hline Gender & & & $0.885^{\mathrm{a}}$ \\
\hline Male & $320(64 \%)$ & $318(64 \%)$ & $0.289^{\mathrm{b}}$ \\
\hline Female & $181(36 \%)$ & $178(36 \%)$ & \\
\hline Age & $61.32 \pm 11.70$ & $60.69 \pm 6.42$ & \\
\hline$>61$ & $250(50 \%)$ & $233(47 \%)$ & \\
$\leq 61$ & $251(50 \%)$ & $263(53 \%)$ & \\
\hline Hypertension & & \\
\hline yes & $296(60 \%)$ & \\
\hline no & $205(40 \%)$ & \\
\hline Diabetes & & \\
\hline yes & $101(20 \%)$ & \\
\hline no & $400(80 \%)$ & \\
\hline${ }^{a} p$ value was calculated from two-sided Chi-squared tests; ${ }^{b} p$ value was calculated from Student's $t$ test. \\
\hline$p<0.05$ indicates statistical significance.
\end{tabular}

\subsection{Association between VEGFA variations and CHD risk.}

In our study, the basic information about the selected SNPs in VEGFA gene was listed in Table 3. The genotype distribution of all candidate SNPs in control group were in accordance with HWE ( $p$ value $>0.05$ ). We had not found that any SNPs were significantly associated with the risk of CHD in the allele model (all $p>0.05$ ). Four genetic models, including the codominant model, the dominant model, the recessive model, and the Log-additive model were carried out to analyze the relationship between SNPs genotypes and CHD risk. The results were showed in Table 4. The significantly positive association was found between the "C/T" of rs3025021 in VEGFA and CHD susceptibility in the codominant model $(\mathrm{OR}=1.35,95 \% \mathrm{Cl}=1.02-1.80, p=0.038)$ after adjustment for gender and age, which showed that the rs3025021 was a risk factor in the development of CHD. However, we observed no significant correlation between other SNPs and CHD risk.

Table 3

Basic information of the 5 SNPs in this study.

\begin{tabular}{|c|c|c|c|c|c|c|c|c|}
\hline \multirow[t]{2}{*}{ SNP-ID } & \multirow[t]{2}{*}{ Chr. } & \multirow[t]{2}{*}{ Position } & \multirow{2}{*}{$\begin{array}{l}\text { Allele } \\
\text { (A/B) }\end{array}$} & \multicolumn{2}{|l|}{ MAF } & \multirow{2}{*}{$p^{\mathrm{a}-\mathrm{HWE}}$} & \multicolumn{2}{|l|}{ Allele model } \\
\hline & & & & Cases & Controls & & OR(95\%Cl) & $p^{b}$ \\
\hline rs833068 & 6 & 43774790 & $\mathrm{~A} / \mathrm{G}$ & 0.411 & 0.440 & 0.412 & $0.89(0.74-1.06)$ & 0.185 \\
\hline rs833070 & 6 & 43774889 & $\mathrm{C} / \mathrm{T}$ & 0.238 & 0.229 & 0.252 & $1.05(0.85-1.30)$ & 0.631 \\
\hline rs3025021 & 6 & 43781426 & $\mathrm{C} / \mathrm{T}$ & 0.168 & 0.145 & 0.204 & $1.19(0.93-1.51)$ & 0.167 \\
\hline rs3025030 & 6 & 43782850 & $\mathrm{C} / \mathrm{G}$ & 0.178 & 0.156 & 0.393 & $1.17(0.92-1.48)$ & 0.200 \\
\hline rs6905288 & 6 & 43791136 & $A / G$ & 0.262 & 0.268 & 0.210 & $0.97(0.8-1.19)$ & 0.774 \\
\hline
\end{tabular}

SNP, single-nucleotide polymorphism; MAF, minor allele frequency; $\mathrm{HWE}$, Hardy-Weinberg equilibrium; OR, odds ratio; $\mathrm{Cl}$, confidence interval. $p<0.05$ indicates statistical significance. 
Table 4

SNPs associated with the CHD with adjustments for gender and age.

\begin{tabular}{|c|c|c|c|c|c|c|c|c|}
\hline \multirow[t]{2}{*}{ SNP } & \multirow[t]{2}{*}{ Model } & \multirow[t]{2}{*}{ Genotype } & \multirow[t]{2}{*}{ Case } & \multirow[t]{2}{*}{ Control } & \multicolumn{2}{|l|}{ Before adjusted } & \multicolumn{2}{|l|}{ After adjusted } \\
\hline & & & & & $\mathrm{OR}(95 \% \mathrm{Cl})$ & $p$-value & $\mathrm{OR}(95 \% \mathrm{Cl})$ & $p$-value \\
\hline \multirow[t]{8}{*}{ rs833068 } & Co-dominant & $\mathrm{G} / \mathrm{G}$ & $175(35.00 \%)$ & $150(30.31 \%)$ & $1[$ Ref] & & $1[$ Ref] & \\
\hline & & $A / G$ & $239(47.80 \%)$ & $254(51.31 \%)$ & $0.81(0.61-1.07)$ & 0.133 & $0.80(0.61-1.07)$ & 0.128 \\
\hline & & $\mathrm{A} / \mathrm{A}$ & $86(17.20 \%)$ & $91(18.38 \%)$ & $0.81(0.56-1.17)$ & 0.260 & $0.81(0.56-1.17)$ & 0.254 \\
\hline & Dominant & $\mathrm{G} / \mathrm{G}$ & $175(35.00 \%)$ & $150(30.31 \%)$ & $1[$ Ref] & & $1[$ Ref] & \\
\hline & & $\mathrm{A} / \mathrm{G}-\mathrm{A} / \mathrm{A}$ & $325(65.00 \%)$ & $345(69.69)$ & $0.81(0.62-1.05)$ & 0.114 & $0.80(0.62-1.05)$ & 0.110 \\
\hline & Recessive & $\mathrm{G} / \mathrm{G}-\mathrm{A} / \mathrm{G}$ & $414(82.80 \%)$ & $404(81.62 \%)$ & $1[$ Ref] & & $1[$ Ref] & \\
\hline & & $A / A$ & $86(17.20 \%)$ & $91(18.38 \%)$ & $0.92(0.67-1.28)$ & 0.625 & $0.92(0.67-1.28)$ & 0.623 \\
\hline & Log-additive & - & - & - & $0.89(0.74-1.06)$ & 0.182 & $0.88(0.74-1.06)$ & 0.177 \\
\hline \multirow[t]{8}{*}{ rs833070 } & Co-dominant & $\mathrm{T} / \mathrm{T}$ & $285(57.34 \%)$ & $289(58.38 \%)$ & $1[$ Ref] & & $1[$ Ref] & \\
\hline & & $\mathrm{C} / \mathrm{T}$ & $187(37.63 \%)$ & $185(37.38 \%)$ & $1.03(0.79-1.33)$ & 0.853 & $1.02(0.79-1.33)$ & 0.881 \\
\hline & & $\mathrm{C} / \mathrm{C}$ & $25(5.03 \%)$ & $21(4.24 \%)$ & $1.21(0.66-2.21)$ & 0.540 & $1.20(0.66-2.19)$ & 0.558 \\
\hline & Dominant & $\mathrm{T} / \mathrm{T}$ & $285(57.34 \%)$ & $289(58.38 \%)$ & $1[$ Ref] & & $1[$ Ref] & \\
\hline & & $\mathrm{C} / \mathrm{T}-\mathrm{C} / \mathrm{C}$ & $212(42.66 \%)$ & $206(41.62 \%)$ & $1.04(0.81-1.34)$ & 0.740 & $1.04(0.81-1.34)$ & 0.770 \\
\hline & Recessive & $\mathrm{T} / \mathrm{T}-\mathrm{C} / \mathrm{T}$ & 472(94.97) & $474(95.76 \%)$ & $1[$ Ref] & & $1[$ Ref] & \\
\hline & & $\mathrm{C} / \mathrm{C}$ & $25(5.03 \%)$ & $21(4.24 \%)$ & $1.20(0.66-2.17)$ & 0.556 & $1.19(0.66-2.15)$ & 0.569 \\
\hline & Log-additive & - & - & - & $1.06(0.85-1.31)$ & 0.623 & $1.05(0.85-1.3)$ & 0.651 \\
\hline \multirow[t]{8}{*}{ rs3025021 } & Co-dominant & $\mathrm{T} / \mathrm{T}$ & $344(68.66 \%)$ & $366(73.79 \%)$ & $1[$ Ref] & & $1[$ Ref] & \\
\hline & & $\mathrm{C} / \mathrm{T}$ & $146(29.14 \%)$ & 116(23.39\%) & $1.34(1.01-1.78)$ & $0.044^{*}$ & $1.35(1.02-1.80)$ & $0.038^{*}$ \\
\hline & & $\mathrm{C} / \mathrm{C}$ & $11(2.20 \%)$ & $14(2.82 \%)$ & $0.84(0.37-1.87)$ & 0.662 & $0.85(0.38-1.89)$ & 0.687 \\
\hline & Dominant & $\mathrm{T} / \mathrm{T}$ & $344(68.66 \%)$ & $366(73.79 \%)$ & $1[$ Ref] & & $1[$ Ref] & \\
\hline & & $\mathrm{C} / \mathrm{T}-\mathrm{C} / \mathrm{C}$ & 157(31.34\%) & $130(26.21 \%)$ & $1.29(0.98-1.69)$ & 0.074 & $1.30(0.99-1.71)$ & 0.063 \\
\hline & Recessive & $\mathrm{T} / \mathrm{T}-\mathrm{C} / \mathrm{T}$ & $490(97.80 \%)$ & 482(97.18\%) & $1[$ Ref] & & $1[$ Ref] & \\
\hline & & $\mathrm{C} / \mathrm{C}$ & $11(2.20 \%)$ & $14(2.82 \%)$ & $0.77(0.35-1.72)$ & 0.528 & $0.78(0.35-1.74)$ & 0.544 \\
\hline & Log-additive & - & - & - & $1.19(0.93-1.51)$ & 0.168 & $1.20(0.94-1.53)$ & 0.147 \\
\hline \multirow[t]{8}{*}{ rs3025030 } & Co-dominant & $\mathrm{G} / \mathrm{G}$ & $339(67.66 \%)$ & $350(70.56 \%)$ & $1[$ Ref] & & $1[$ Ref] & \\
\hline & & $\mathrm{C} / \mathrm{G}$ & $146(29.14 \%)$ & $137(27.62 \%)$ & $1.10(0.83-1.45)$ & 0.499 & $1.10(0.83-1.45)$ & 0.507 \\
\hline & & $\mathrm{C} / \mathrm{C}$ & $16(3.19 \%)$ & $9(1.81 \%)$ & $1.84(0.80-4.21)$ & 0.152 & $1.81(0.79-4.15)$ & 0.164 \\
\hline & Dominant & $\mathrm{G} / \mathrm{G}$ & $339(67.66 \%)$ & $350(70.56 \%)$ & $1[$ Ref] & & $1[$ Ref] & \\
\hline & & $\mathrm{C} / \mathrm{G}-\mathrm{C} / \mathrm{C}$ & $162(32.34 \%)$ & $146(29.43 \%)$ & $1.15(0.88-1.50)$ & 0.322 & $1.14(0.87-1.50)$ & 0.333 \\
\hline & Recessive & $\mathrm{G} / \mathrm{G}-\mathrm{A} / \mathrm{G}$ & $485(96.81 \%)$ & $487(98.19 \%)$ & $1[$ Ref] & & $1[$ Ref] & \\
\hline & & $\mathrm{C} / \mathrm{C}$ & $16(3.19 \%)$ & $9(1.81 \%)$ & $1.79(0.78-4.08)$ & 0.169 & $1.76(0.77-4.02)$ & 0.182 \\
\hline & Log-additive & - & - & - & $1.17(0.92-1.49)$ & 0.196 & $1.17(0.92-1.48)$ & 0.208 \\
\hline \multirow[t]{7}{*}{ rs6905288 } & Co-dominant & $\mathrm{G} / \mathrm{G}$ & $280(55.89 \%)$ & $260(52.42 \%)$ & $1[$ Ref] & & $1[$ Ref] & \\
\hline & & $A / G$ & $179(35.73 \%)$ & $206(41.53 \%)$ & $0.81(0.62-1.05)$ & 0.108 & $0.81(0.62-1.05)$ & 0.105 \\
\hline & & $\mathrm{A} / \mathrm{A}$ & $42(8.38 \%)$ & $30(6.05 \%)$ & $1.30(0.79-2.14)$ & 0.302 & $1.30(0.79-2.15)$ & 0.296 \\
\hline & Dominant & $\mathrm{G} / \mathrm{G}$ & $280(55.89 \%)$ & $260(52.42 \%)$ & $1[$ Ref] & & $1[$ Ref] & \\
\hline & & $\mathrm{A} / \mathrm{G}-\mathrm{A} / \mathrm{A}$ & $221(44.11 \%)$ & $236(47.58 \%)$ & $0.87(0.68-1.12)$ & 0.272 & $0.87(0.68-1.12)$ & 0.268 \\
\hline & Recessive & $\mathrm{G} / \mathrm{G}-\mathrm{A} / \mathrm{G}$ & $459(91.62 \%)$ & $466(93.95 \%)$ & $1[$ Ref] & & $1[$ Ref] & \\
\hline & & $\mathrm{A} / \mathrm{A}$ & $42(8.38 \%)$ & $30(6.05 \%)$ & $1.42(0.87-2.31)$ & 0.156 & $1.43(0.88-2.32)$ & 0.152 \\
\hline
\end{tabular}




\begin{tabular}{|c|c|c|c|c|c|c|c|c|}
\hline \multirow[t]{2}{*}{ SNP } & \multirow[t]{2}{*}{ Model } & \multirow[t]{2}{*}{ Genotype } & \multirow[t]{2}{*}{ Case } & \multirow[t]{2}{*}{ Control } & \multicolumn{2}{|l|}{ Before adjusted } & \multicolumn{2}{|l|}{ After adjusted } \\
\hline & & & & & $\mathrm{OR}(95 \% \mathrm{Cl})$ & $p$-value & $\mathrm{OR}(95 \% \mathrm{Cl})$ & $p$-value \\
\hline & Log-additive & - & - & - & $0.97(0.80-1.18)$ & 0.775 & $0.97(0.80-1.18)$ & 0.775 \\
\hline
\end{tabular}

\subsection{Stratification analysis by age and gender.}

According to gender and age parameters, stratified analysis regarding the effects of SNPs on CHD risk was summarized in Table 5. The stratification analysis by age adjusted for age and gender showed that rs 833068 in VEGFA was observed to be associated with the reduced risk of CHD at age $>61$ years in the codominant model $(\mathrm{OR}=0.64,95 \% \mathrm{Cl}=0.42-0.98, p=0.042$ for the "A/G" genotype). Meanwhile, the rs 833068 was also associated with the reduced risk of $\mathrm{CHD}$ at age $\leq 61$ years in the recessive model $(\mathrm{OR}=0.58,95 \% \mathrm{Cl}=0.34-0.98, p=0.043)$. In addition, the stratification analysis by gender adjusted for age found that three loci (rs833068, rs3025021 and rs6905288) in VEGFA were related to the CHD risk in males. The rs 833068 was associated with reduced risk of $\mathrm{CHD}$ in the allele model (adjusted, $\mathrm{OR}=0.75,95 \% \mathrm{Cl}=0.01-0.75, p=0.010$ ), the codominant model (adjusted, $\mathrm{OR}=0.58,95 \% \mathrm{Cl}=0.37-0.92, p=0.020 \mathrm{for}$ "A/A" genotype; $\mathrm{OR}=0.65,95 \% \mathrm{Cl}=0.45-0.92, p=0.016$ for "A/G" genotype), the dominant model $(\mathrm{OR}=0.63,95 \% \mathrm{Cl}=0.45-0.88, p=0.006)$, the log-additive model $(\mathrm{OR}=0.74,95 \% \mathrm{Cl}=0.60-0.93, p=0.010)$. The rs3025021 was associated with increased risk of $\mathrm{CHD}$ in the codominant model (adjusted, OR $=1.49$, $95 \% \mathrm{Cl}=1.04-2.13, p=0.029$ for " $\mathrm{C} / \mathrm{T}$ " genotype), the dominant model $(\mathrm{OR}=1.43,95 \% \mathrm{Cl}=1.01-2.02, p=0.041)$. The rs 6905288 was associated with increased risk of $\mathrm{CHD}$ in the recessive model $(\mathrm{OR}=2.03,95 \% \mathrm{Cl}=1.08-3.78, p=0.027)$. However, we found no any genotypes of the selected SNPs were associated with $\mathrm{CHD}$ in females in any genetic model. 
Table 5

Stratified analysis on association between selected SNPs and CHD risk.

\begin{tabular}{|c|c|c|c|c|c|c|c|}
\hline & \multirow[t]{2}{*}{ SNP ID } & \multicolumn{6}{|l|}{$p^{\dagger}, \mathrm{OR}(95 \% \mathrm{Cl})$} \\
\hline & & Allele & Homozygote & Heterozygote & Dominant & Recessive & Additive \\
\hline \multicolumn{8}{|l|}{ Age } \\
\hline$>61$ & rs833068 & $\begin{array}{l}0.219,0.86(0.67- \\
1.10)\end{array}$ & $\begin{array}{l}0.875,0.96(0.56- \\
1.64)\end{array}$ & $\begin{array}{l}0.042^{*}, 0.64(0.42- \\
0.98)\end{array}$ & $\begin{array}{l}0.105,0.72(0.48- \\
1.07)\end{array}$ & $\begin{array}{l}0.343,1.26(0.78- \\
2.02)\end{array}$ & $\begin{array}{l}0.586,0.93(0.71- \\
1.21)\end{array}$ \\
\hline$\leq 61$ & rs833068 & $\begin{array}{l}0.551,0.92(0.72- \\
1.20)\end{array}$ & $\begin{array}{l}0.150,0.65(0.36- \\
1.17)\end{array}$ & $\begin{array}{l}0.375,1.22(0.79- \\
1.88)\end{array}$ & $\begin{array}{l}0.851,1.04(0.69- \\
1.57)\end{array}$ & $\begin{array}{l}0.043^{*}, 0.58(0.34- \\
0.98)\end{array}$ & $\begin{array}{l}0.335,0.87(0.66- \\
1.15)\end{array}$ \\
\hline \multicolumn{8}{|l|}{ Gender } \\
\hline \multirow[t]{3}{*}{ Male } & rs833068 & $\begin{array}{l}0.010^{*}, 0.75(0.01- \\
0.75)\end{array}$ & $\begin{array}{l}0.020^{*}, 0.58(0.37- \\
0.92)\end{array}$ & $\begin{array}{l}0.016^{*}, 0.65(0.45- \\
0.92)\end{array}$ & $\begin{array}{l}0.006^{*}, 0.63(0.45- \\
0.88)\end{array}$ & $\begin{array}{l}0.172,0.76(0.50- \\
1.13)\end{array}$ & $\begin{array}{l}0.010^{*}, 0.74(0.60- \\
0.93)\end{array}$ \\
\hline & rs3025021 & $\begin{array}{l}0.087,1.30(0.09- \\
1.30)\end{array}$ & $\begin{array}{l}0.966,0.98(0.37- \\
2.58)\end{array}$ & $\begin{array}{l}0.029^{*}, 1.49(1.04- \\
2.13)\end{array}$ & $\begin{array}{l}0.041^{*}, 1.43(1.01- \\
2.02)\end{array}$ & $\begin{array}{l}0.796,0.88(0.34- \\
2.31)\end{array}$ & $\begin{array}{l}0.089,1.3(0.96- \\
1.76)\end{array}$ \\
\hline & rs6905288 & $\begin{array}{l}0.449,1.10(0.45- \\
1.10)\end{array}$ & $\begin{array}{l}0.05,1.89(1.00- \\
3.59)\end{array}$ & $\begin{array}{l}0.332,0.85(0.61- \\
1.18)\end{array}$ & $\begin{array}{l}0.817,0.96(0.71- \\
1.32)\end{array}$ & $\begin{array}{l}0.027^{*}, 2.03(1.08- \\
3.78)\end{array}$ & $\begin{array}{l}0.453,1.1(0.86- \\
1.41)\end{array}$ \\
\hline \multirow[t]{3}{*}{ Female } & rs833068 & $\begin{array}{l}0.213,1.21(0.90- \\
1.62)\end{array}$ & $\begin{array}{l}0.227,1.48(0.79- \\
2.77)\end{array}$ & $\begin{array}{l}0.506,1.17(0.73- \\
1.87)\end{array}$ & $\begin{array}{l}0.344,1.24(0.79- \\
1.94)\end{array}$ & $\begin{array}{l}0.309,1.34(0.76- \\
2.35)\end{array}$ & $\begin{array}{l}0.228,1.21(0.89- \\
1.64)\end{array}$ \\
\hline & rs3025021 & $\begin{array}{l}0.993,1.00(0.67- \\
1.51)\end{array}$ & $\begin{array}{l}0.622,0.69(0.16- \\
2.99)\end{array}$ & $\begin{array}{l}0.63,1.13(0.70- \\
1.81)\end{array}$ & $\begin{array}{l}0.734,1.08(0.68- \\
1.72)\end{array}$ & $\begin{array}{l}0.591,0.67(0.16- \\
2.88)\end{array}$ & $\begin{array}{l}0.885,1.03(0.68- \\
1.56)\end{array}$ \\
\hline & rs6905288 & $\begin{array}{l}0.137,0.78(0.56- \\
1.08)\end{array}$ & $\begin{array}{l}0.341,0.66(0.29- \\
1.54)\end{array}$ & $\begin{array}{l}0.161,0.73(0.47- \\
1.13)\end{array}$ & $\begin{array}{l}0.125,0.72(0.47- \\
1.10)\end{array}$ & $\begin{array}{l}0.508,0.76(0.33- \\
1.72)\end{array}$ & $\begin{array}{l}0.135,0.77(0.55- \\
1.08)\end{array}$ \\
\hline \multicolumn{8}{|c|}{ Hypertension } \\
\hline yes & rs3025021 & $\begin{array}{l}0.088,1.27(0.96- \\
1.67)\end{array}$ & $\begin{array}{l}0.824,1.11(0.45- \\
2.69)\end{array}$ & $\begin{array}{l}0.029^{*}, 1.44(1.04- \\
2.00)\end{array}$ & $\begin{array}{l}0.035^{*}, 1.41(1.02- \\
1.93)\end{array}$ & $\begin{array}{l}0.999,1.00(0.41- \\
2.42)\end{array}$ & $\begin{array}{l}0.067,1.29(0.98- \\
1.70)\end{array}$ \\
\hline \multirow[t]{3}{*}{ no } & rs833068 & $\begin{array}{l}0.046,0.79(0.62- \\
1.00)\end{array}$ & $\begin{array}{l}0.037^{*}, 0.58(0.35- \\
0.97)\end{array}$ & $\begin{array}{l}0.212,0.79(0.55- \\
1.14)\end{array}$ & $\begin{array}{l}0.084,0.74(0.52- \\
1.04)\end{array}$ & $\begin{array}{l}0.087,0.67(0.42- \\
1.06)\end{array}$ & $\begin{array}{l}0.034^{*}, 0.77(0.60- \\
0.98)\end{array}$ \\
\hline & rs3025030 & $\begin{array}{l}0.047,1.35(1.00- \\
1.82)\end{array}$ & $\begin{array}{l}0.041^{*}, 2.7(1.04- \\
6.99)\end{array}$ & $\begin{array}{l}0.206,1.26(0.88- \\
1.81)\end{array}$ & $\begin{array}{l}0.090,1.35(0.95- \\
1.91)\end{array}$ & $\begin{array}{l}0.055,2.52(0.98- \\
6.47)\end{array}$ & $\begin{array}{l}0.037^{*}, 1.38(1.02- \\
1.87)\end{array}$ \\
\hline & rs6905288 & $\begin{array}{l}0.930,0.99(0.76- \\
1.28)\end{array}$ & $\begin{array}{l}0.102,1.64(0.91- \\
2.97)\end{array}$ & $\begin{array}{l}0.051,0.7(0.49- \\
1.00)\end{array}$ & $\begin{array}{l}0.241,0.82(0.59- \\
1.14)\end{array}$ & $\begin{array}{l}0.031^{*}, 1.89(1.06- \\
3.37)\end{array}$ & $\begin{array}{l}0.983,1.00(0.77- \\
1.29)\end{array}$ \\
\hline \multicolumn{8}{|c|}{ Diabetes } \\
\hline yes & rs3025021 & $\begin{array}{l}0.025^{*}, 1.55(1.05- \\
2.27)\end{array}$ & $\begin{array}{l}0.928,0.93(0.20- \\
4.25)\end{array}$ & $\begin{array}{l}0.002^{*}, 2.06(1.30- \\
3.27)\end{array}$ & $\begin{array}{l}0.004^{*}, 1.94(1.24- \\
3.05)\end{array}$ & $\begin{array}{l}0.704,0.75(0.17- \\
3.37)\end{array}$ & $\begin{array}{l}0.016,1.60(1.09- \\
2.37)\end{array}$ \\
\hline no & rs833068 & $\begin{array}{l}0.130,0.86(0.72- \\
1.04)\end{array}$ & $\begin{array}{l}0.246,0.80(0.54- \\
1.17)\end{array}$ & $\begin{array}{l}0.035^{*}, 0.73(0.54- \\
0.98)\end{array}$ & $\begin{array}{l}0.039^{*}, 0.75(0.56- \\
0.99)\end{array}$ & $\begin{array}{l}0.821,0.96(0.68- \\
1.36)\end{array}$ & $\begin{array}{l}0.130,0.86(0.72- \\
1.04)\end{array}$ \\
\hline
\end{tabular}

SNP: single-nucleotide polymorphism; OR: odds ratio; Cl: confidence interval; CHD: coronary heart disease.

$p<0.05$ indicates statistical significance.

\subsection{Stratification analysis with/without hypertension or diabetes.}

We next analyzed the relationship between selected SNPs and CHD-associated diseases, which include hypertension and diabetes. In CHD patients with hypertension, we found that rs3025021 was associated with increased risk of $\mathrm{CHD}$ in the codominant model (adjusted, $\mathrm{OR}=1.44,95 \% \mathrm{Cl}=1.04-2.00, p=$ 0.029 for " $\mathrm{C} / \mathrm{T}$ " genotype), the dominant model $(\mathrm{OR}=1.41,95 \% \mathrm{Cl}=1.012-1.93, p=0.035)$. Conversely, in the CHD patients without hypertension, three loci (rs833068, rs3025030 and rs6905288) in VEGFA were related to the CHD risk. The rs833068 was associated with reduced risk of CHD in the codominant model (adjusted, $\mathrm{OR}=0.58,95 \% \mathrm{Cl}=0.35-0.97, p=0.037$ for "A/A" genotype), the log-additive model $(\mathrm{OR}=0.77,95 \% \mathrm{Cl}=0.60-0.98, p=0.034)$. The rs3025030 was associated with increased risk of CHD in the codominant model (adjusted, OR $=2.70,95 \% \mathrm{Cl}=1.04-6.99, p=0.041$ for "C/C" genotype), the log-additive model $(\mathrm{OR}=1.38,95 \% \mathrm{Cl}=1.02-1.87, p=0.037)$. The rs6905288 was associated with increased risk of $\mathrm{CHD}$ in the recessive model (adjusted, OR $=1.89,95 \% \mathrm{Cl}=1.06-3.37, p=0.031)$.

In $\mathrm{CHD}$ patients with diabetes, we found that rs3025021 was associated with increased risk of $\mathrm{CHD}$ in the allele model (adjusted, $\mathrm{OR}=1.55,95 \% \mathrm{Cl}=1.05-$ 2.27, $p=0.025$ ), the codominant model (adjusted, $\mathrm{OR}=2.06,95 \% \mathrm{Cl}=1.30-3.27, p=0.002$ for "C/T" genotype), the dominant model $(\mathrm{OR}=1.94,95 \% \mathrm{Cl}=1.24-$ $3.05, p=0.004)$. In CHD patients without diabetes, the rs 833068 was associated with reduced risk of $\mathrm{CHD}$ in the codominant model (adjusted, $\mathrm{OR}=0.73,95 \%$ $\mathrm{Cl}=0.54-0.98, p=0.035$ for " $\mathrm{A} / \mathrm{G}$ " genotype), the dominant model $(\mathrm{OR}=0.75,95 \% \mathrm{Cl}=0.56-0.99, p=0.039)$.

\subsection{Haplotype association}


Finally, the LD block and haplotype analyses of the selected SNPs in all subjects were further studied. And we found a strong LD between rs833068 and rs833070 with $D^{\prime}=1.00$ (Fig. 1). The results of the association between haplotypes and the CHD risk were shown in Table 6 . However, we found no haplotypes were associated with the risk of $\mathrm{CHD}$ even if after logistic regression analysis adjustment for age and gender.

Table 6

Haplotype analysis results in this study.

\begin{tabular}{|c|c|c|c|c|c|c|}
\hline \multirow[t]{2}{*}{ Haplotype } & \multicolumn{2}{|c|}{ Frequency } & \multicolumn{2}{|l|}{ Before adjusted } & \multicolumn{2}{|l|}{ After adjusted } \\
\hline & Case & Control & $\mathrm{OR}(95 \% \mathrm{Cl})$ & $p$-value & $\mathrm{OR}(95 \% \mathrm{Cl})$ & $p^{\mathrm{a}}$-value \\
\hline \multicolumn{7}{|c|}{ rs833068|rs833070 } \\
\hline GT & 0.239 & 0.230 & $1.06(0.85-1.31)$ & 0.622 & $1.05(0.85-1.30)$ & 0.651 \\
\hline$A C$ & 0.412 & 0.439 & $0.89(0.75-1.07)$ & 0.221 & $0.89(0.75-1.07)$ & 0.215 \\
\hline $\mathrm{GC}$ & 0.651 & 0.669 & $0.92(0.77-1.11)$ & 0.407 & $0.92(0.76-1.11)$ & 0.378 \\
\hline \multicolumn{7}{|c|}{ SNP: single-nucleotide polymorphism; OR: odds ratio; Cl: confidence interval. } \\
\hline$P^{\mathrm{a}}:$ Adjust & and a & & & & & \\
\hline
\end{tabular}

\section{Discussion}

In this case-control study, allele, genotype and haplotype frequencies of five SNPs in the VEGFA gene between CHD patients and healthy controls were compared and stratification analyses were conducted. The genotype"C/T" of rs3025021 in VEGFA was found to be associated with CHD susceptibility in the codominant model (adjusted, $\mathrm{OR}=1.35,95 \% \mathrm{Cl}=1.02-1.80, p=0.038$ ) in the overall. The stratification analysis by age showed that rs 833068 in $V E G F A$ were observed to be associated with the reduced risk of $\mathrm{CHD}$ at age $>61$ years and age $\leq 61$ years, respectively. And the stratification analysis by gender found that three loci (rs833068, rs3025021 and rs6905288) in VEGFA were related to the CHD risk in males. In addition, we found that rs3025021 was associated with increased risk of CHD in patients with hypertension. Conversely, three loci (rs833068, rs3025030 and rs6905288) in VEGFA were related to the CHD risk in patients without hypertension. Furthermore, rs3025021 was associated with increased risk of CHD in patients with diabetes. The rs833068 was associated with reduced risk of CHD in patients without diabetes. Finally, we found a strong LD between rs 833068 and rs833070. To our knowledge, this is the first study that evaluate and show an association of VEGFA genetic variants with risk of developing CHD in Han Chinese population.

Coronary heart disease (CHD) is a common and complicated cardiovascular disease in the worldwide, and caused by narrowing of the coronary arteries and a lack of blood supply (12). However, the etiological factors for CHD are not fully understood. Like lung cancer (19) and glioma (20), it's clear that genetic factors, such as single nucleotide polymorphism (SNPs) may also play a pivotal role in determining susceptibility of CHD (21). VEGF can not only promote the vascular recanalization and establishment of collateral circulation, but also enhance the dependent vasodilatation of endothelial cells which are closely related to coronary heart disease (22). Several studies have shown that appropriate timing and dose of VEGF is essential to avoid cardiovascular defects during heart development $(23,24)$. VEGFA, as a member of $V E G F$, has been proved to promote the differentiation, proliferation and migration of microvascular endothelial cells by binding to their receptors, thus improving the formation and development (24). In addition, the study has shown that VEGFA may play an important role in the process of epithelial-mesenchymal transformation (EMT) and regulate the formation of endocardial cushion (22).

Recently, several studies have explored the association between the SNPs in VEGFA and the susceptibility to CHD $(12,25,26)$. Han et al $(25)$ showed that rs3025039 in VEGFA gene was remarkably associated with CHD risk in Han Chinese population. On the contrary, Griffin et al (26) conducted a meta-analysis which demonstrated VEGFA polymorphisms may not be associated with CHD. Furthermore, Dong et al (12) found three SNPs (rs699947, rs3025039, and rs 1570360) were remarkably correlated with the susceptibility to CHD. To further clarify the relationship between SNPs within VEGFA and CHD,we genotyped five SNPs (rs833068, rs833070, rs3025021, rs3025030, rs6905288) in this study and discovered that all the five SNPs were significantly with the risk of CHD.

Inevitably, several limitations should be addressed with regard to the case-control study. First, our sample was limited to the Han Chinese population, thus the investigation results might not be applicable to other Chinese populations or additional ethnic groups. Larger and more diversity sample is needed to identify the role of VEGFA genetic polymorphisms in CHD risk. Besides, it could not be ignored that the uniqueness of samples might exaggerate the correlation between the selected 5 SNPs and CHD risk, or neglect the role of some other vital polymorphisms in VEGFA in susceptibility to CHD. Therefore, large prospective cohort studies or combined meta-analyses should be designed to address these limitations.

\section{Conclusion}

In summary, the present study indicated that gene polymorphisms in VEGFA gene were notably correlated with altered CHD risk in the Han Chinese population. Thus, the VEGFA mutations might be used to clinical medicine as genetic marker.

\section{Declarations}

\section{Acknowledgement}

We thank all of the participants for their involvement in this study. We are very grateful to the clinicians and other hospital staff for providing blood samples and data collection for this study. 


\section{Authors' contributions}

Conceived and designed the experiments: Shijuan Lu. Performed the experiments: Kang Huang, Yilei Zhou. Analyzed the data: Dehong Lin, Zibin Chen. Contributed reagents/materials/analysis tools: Zanrui Zhong. Wrote the paper: Jianghua Zhong. Revised囚Shijuan Lu.

\section{Funding}

This study was supported by Hainan Natural Science Foundation Project (No. 20168320.00).

\section{Competing interests}

None

\section{References}

1. Pfisterer ME, Zellweger MJ, Gersh BJ. Management of stable coronary artery disease. Lancet (London, England). 2010;375(9716):763-72. doi: 10.1016/s0140-6736(10)60168-7.

2. Ross R. Atherosclerosis-an inflammatory disease. The New England journal of medicine. 1999;340(2):115-26. doi: 10.1056/nejm199901143400207.

3. Heusch G, Libby P, Gersh B, Yellon D, Bohm M, Lopaschuk G, et al. Cardiovascular remodelling in coronary artery disease and heart failure. Lancet (London, England). 2014;383(9932):1933-43. doi: 10.1016/s0140-6736(14)60107-0.

4. Walfridsson $\mathrm{H}$, Lund $\mathrm{N}$. Tissue oxygen pressure in normal myocardium and across the border zone during coronary artery occlusion in the pig. Effects of different arterial oxygen pressures. Basic research in cardiology. 1990;85(5):467-80.

5. Lloyd-Jones D, Adams R, Carnethon M, De Simone G, Ferguson TB, Flegal K, et al. Heart disease and stroke statistics-2009 update: a report from the American Heart Association Statistics Committee and Stroke Statistics Subcommittee. Circulation. 2009;119(3):480-6. doi: 10.1161/circulationaha.108.191259.

6. Chen BJ, Pan ZQ, Su XX. [Study on changes of TCM syndrome in patients with coronary heart disease before and after intervention treatment]. Zhongguo Zhong xi yi jie he za zhi Zhongguo Zhongxiyi jiehe zazhi = Chinese journal of integrated traditional and Western medicine. 2007;27(8):689-91.

7. Pogosova N, Kotseva K, De Bacquer D, von Kanel R, De Smedt D, Bruthans J, et al. Psychosocial risk factors in relation to other cardiovascular risk factors in coronary heart disease: Results from the EUROASPIRE IV survey. A registry from the European Society of Cardiology. European journal of preventive cardiology. 2017;24(13):1371-80. doi: 10.1177/2047487317711334.

8. Peyser PA. Genetic epidemiology of coronary artery disease. Epidemiologic reviews. 1997;19(1):80-90. doi: 10.1093/oxfordjournals.epirev.a017949.

9. Xiao L, Shi D, Zhang H, Zhang Y, Liu Y, Lu H, et al. Association between single nucleotide polymorphism rs11057401 of CCDC92 gene and the risk of coronary heart disease (CHD). Lipids in health and disease. 2018;17(1):28. doi: 10.1186/s12944-018-0672-1.

10. Kanu JS, Gu Y, Zhi S, Yu M, Lu Y, Cong Y, et al. Single nucleotide polymorphism rs3774261 in the AdipoQ gene is associated with the risk of coronary heart disease (CHD) in Northeast Han Chinese population: a case-control study. Lipids in health and disease. 2016;15:6. doi: 10.1186/s12944-015-0173-4.

11. Vincenti V, Cassano C, Rocchi M, Persico G. Assignment of the vascular endothelial growth factor gene to human chromosome 6p21.3. Circulation. 1996;93(8):1493-5.

12. Liu D, Song J, Ji X, Liu Z, Cong M, Hu B. Association of Genetic Polymorphisms on VEGFA and VEGFR2 With Risk of Coronary Heart Disease. Medicine. 2016;95(19):e3413. doi: 10.1097/md.0000000000003413.

13. Chen HL, Liu K. Vascular endothelial growth factor polymorphisms and coronary artery disease: a systemic review and meta-analysis. International journal of cardiology. 2014;172(1):e220-4. doi: 10.1016/j.ijcard.2013.12.156.

14. Geng TT, Xun XJ, Li S, Feng T, Wang LP, Jin TB, et al. Association of colorectal cancer susceptibility variants with esophageal cancer in a Chinese population. World journal of gastroenterology. 2015;21(22):6898-904. doi: 10.3748/wjg.v21.i22.6898.

15. Gabriel S, Ziaugra L, Tabbaa D. SNP genotyping using the Sequenom MassARRAY iPLEX platform. Current protocols in human genetics. 2009;Chapter 2:Unit 2.12. doi: 10.1002/0471142905.hg0212s60.

16. Thomas RK, Baker AC, Debiasi RM, Winckler W, Laframboise T, Lin WM, et al. High-throughput oncogene mutation profiling in human cancer. Nature genetics. 2007;39(3):347-51. doi: 10.1038/ng1975.

17. Bland JM, Altman DG. Statistics notes. The odds ratio. BMJ (Clinical research ed). 2000;320(7247):1468. doi: 10.1136/bmj.320.7247.1468.

18. Clarke GM, Anderson CA, Pettersson FH, Cardon LR, Morris AP, Zondervan KT. Basic statistical analysis in genetic case-control studies. Nature protocols 2011;6(2):121-33. doi: 10.1038/nprot.2010.182.

19. Gao L, Thakur A, Liang Y, Zhang S, Wang T, Chen T, et al. Polymorphisms in the TERT gene are associated with lung cancer risk in the Chinese Han population. European journal of cancer prevention : the official journal of the European Cancer Prevention Organisation (ECP). 2014;23(6):497-501. doi: 10.1097/cej.0000000000000086.

20. Li G, Jin TB, Wei XB, He SM, Liang HJ, Yang HX, et al. Selected polymorphisms of GSTP1 and TERT were associated with glioma risk in Han Chinese. Cancer epidemiology. 2012;36(6):525-7. doi: 10.1016/j.canep.2012.06.008.

21. Song C, Chang Z, Magnusson PK, Ingelsson E, Pedersen NL. Genetic factors may play a prominent role in the development of coronary heart disease dependent on important environmental factors. Journal of internal medicine. 2014;275(6):631-9. doi: 10.1111/joim.12177. 
22. Wang E, Wang Z, Liu S, Gu H, Gong D, Hua K, et al. Polymorphisms of VEGF, TGFbeta1, TGFbetaR2 and conotruncal heart defects in a Chinese population. Molecular biology reports. 2014;41(3):1763-70. doi: 10.1007/s11033-014-3025-9.

23. Miquerol L, Langille BL, Nagy A. Embryonic development is disrupted by modest increases in vascular endothelial growth factor gene expression. Development (Cambridge, England). 2000;127(18):3941-6.

24. Carmeliet P, Ferreira V, Breier G, Pollefeyt S, Kieckens L, Gertsenstein M, et al. Abnormal blood vessel development and lethality in embryos lacking a single VEGF allele. Nature. 1996;380(6573):435-9. doi: 10.1038/380435a0.

25. Han X, Liu L, Niu J, Yang J, Zhang Z, Zhang Z. Association between VEGF polymorphisms (936c/t, $-460 \mathrm{t} / \mathrm{c}$ and $-634 \mathrm{~g} / \mathrm{c})$ with haplotypes and coronary heart disease susceptibility. International journal of clinical and experimental pathology. 2015;8(1):922-7.

26. Griffin HR, Hall DH, Topf A, Eden J, Stuart AG, Parsons J, et al. Genetic variation in VEGF does not contribute significantly to the risk of congenital cardiovascular malformation. PloS one. 2009;4(3):e4978. doi: 10.1371/journal.pone.0004978.

\section{Figures}

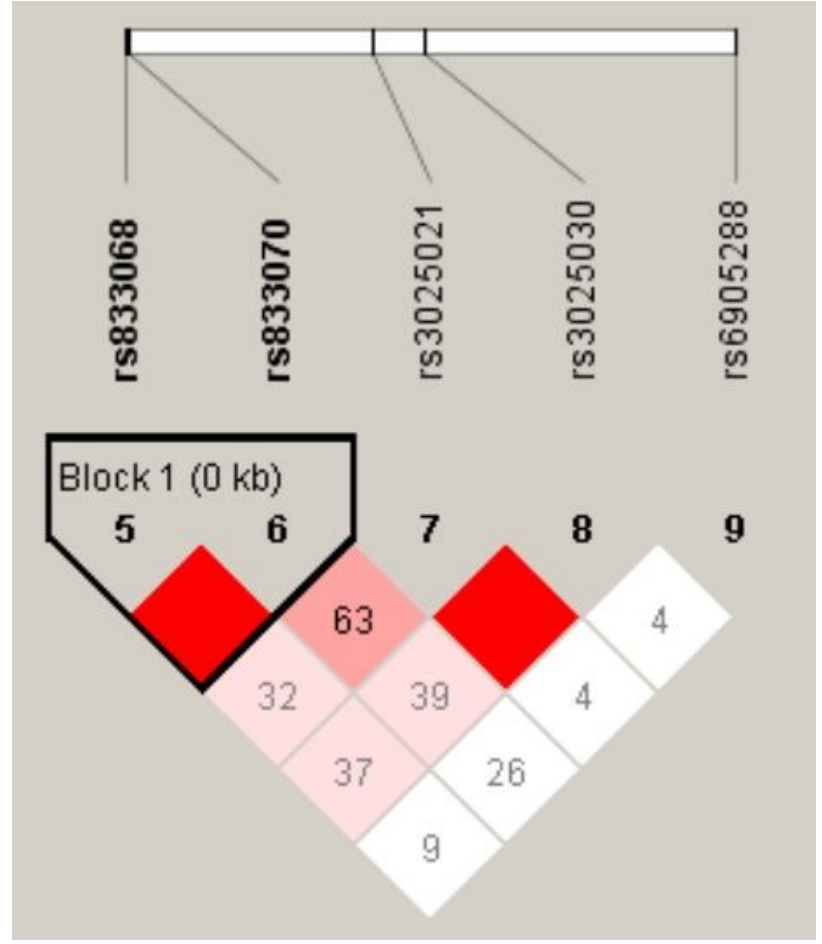

Figure 1

The LD block and haplotype analyses of the selected SNPs in all subjects were further studied. And we found a strong LD between rs833068 and rs833070 with $D^{\prime}=1.00$ (Figure 1). 


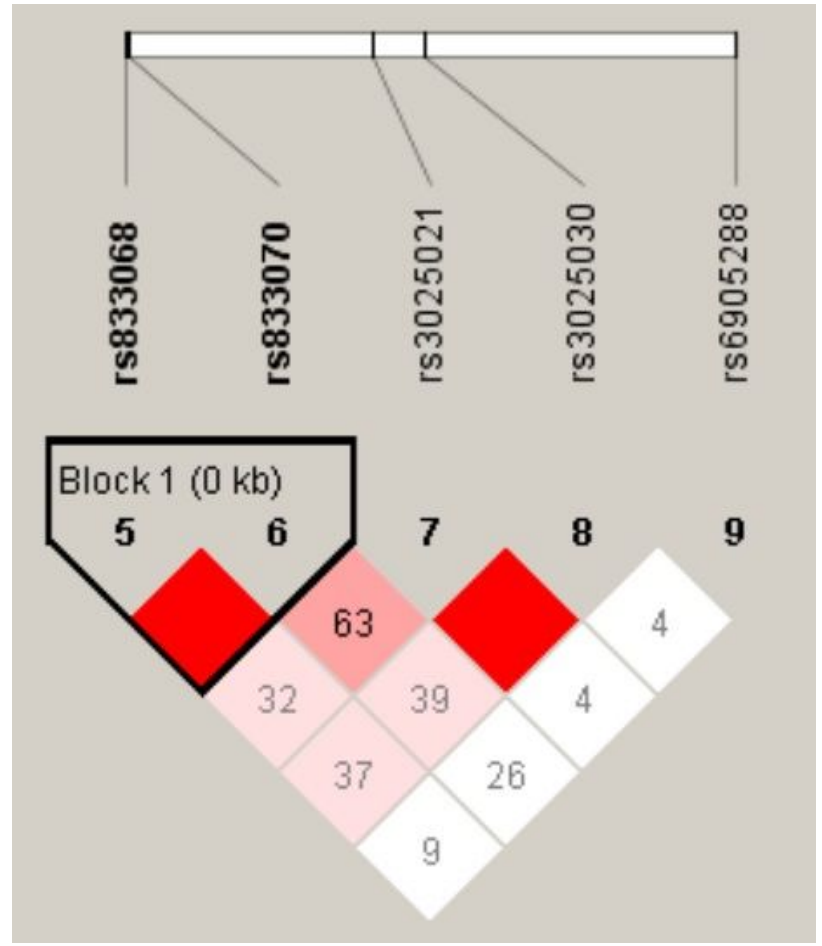

Figure 1

The LD block and haplotype analyses of the selected SNPs in all subjects were further studied. And we found a strong LD between rs833068 and rs833070 with $D^{\prime}=1.00$ (Figure 1). 